\title{
Coherent Detection Method Using DSP for Demodulation of Signal and Subsequent Equalization of Propagation Impairments
}

\author{
Michael G. Taylor, Member, IEEE
}

\begin{abstract}
A new approach to coherent detection is demonstrated which achieves the same high sensitivity as homodyne detection but without the need to phase lock the local oscillator laser. In addition, $1470 \mathrm{ps} / \mathrm{nm}$ of chromatic dispersion is compensated with zero net penalty by electronic domain equalization, a result which has not been achieved before because zero-penalty equalization is not possible after direct detection. The method proposes the use of high-speed digital signal processing technology, and the experimental results are obtained using burst-mode sampling followed by offline signal processing.
\end{abstract}

Index Terms-Chromatic dispersion (CD) compensation, coherent detection, optical transmission, phase diverse detection.

\section{INTRODUCTION}

C OHERENT detection has many advantages over direct detection, which is why it was the subject of much research activity ten years ago. It is sensitive to the phase as well as the amplitude of the optical wave, and coherent detection offers an inherent ultranarrow optical filtering capability useful for dense wavelength-division multiplexing [1]. The mode of coherent detection that gives the best sensitivity [lowest bit-error rate at a given optical signal-to-noise ratio (OSNR)] is homodyne detection, but this mode requires the use of special narrow linewidth lasers that are phase locked, which makes it expensive to implement. This letter proposes a new method of coherent detection based on phase diverse detection and using new high-speed digital signal processing (DSP) technology. It possesses all the advantages of homodyne detection but without the need to phase lock lasers, and so will be much more cost effective. The reported experimental results show that the method can detect a 10-Gb/s signal with very good sensitivity. DSP technology operating at $10 \mathrm{~Gb} / \mathrm{s}$ has very recently been applied to soft decision forward-error correction [2], and as a means to overcome distortions from chromatic dispersion (CD) and polarization-mode dispersion in single-mode fiber (SMF) and modal dispersion in multimode fiber [3]. To date, all proposals for impairment compensation have been to use DSP in conjunction with direct detection. The detected signal is digitized by an analog-to-digital (A/D) converter operating at the bit rate, and then passed to a digital integrated circuit which applies a mathematical algorithm operating on near-neighbor samples to reverse any distortion that the signal experienced

Manuscript received May 6, 2003; revised September 11, 2003.

The author is with the Optical Networks Group, Department of Electronic and Electrical Engineering, University College London, London WC1E 7JE, U.K. (e-mail: mtaylor@unodos.net).

Digital Object Identifier 10.1109/LPT.2003.823106 earlier in the optical domain. Kanter et al. [3] have demonstrated compensation for the CD of $75 \mathrm{~km}$ of SMF at $10 \mathrm{~Gb} / \mathrm{s}$, but the penalty was reduced rather than eliminated. This will always be the case with CD compensation following direct detection, because the phase information is discarded upon detection and it is not possible to restore the signal to its original state. In this letter, penalty-free compensation of $\mathrm{CD}$ is demonstrated for the first time by equalization after coherent detection.

\section{THEORY}

Coherent detection involves beating an incoming signal with light from a local oscillator (LO) laser [1]. With phase diverse detection both the signal and LO are split in two paths and then combined with one another before being detected by two photodetectors [4], as shown in the diagram of the experimental arrangement in Fig. 1. The LO receives an extra phase shift of $\pi / 2$ in one arm. If the signal is written in complex form as $E_{s}(t) \exp \left(i \omega_{s} t\right)$, and the LO as $E_{\mathrm{LO}} \exp \left(i \omega_{\mathrm{LO}} t\right)$, then the powers in the two arms are

$$
\begin{aligned}
& P_{1}(t)=\left|E_{\mathrm{LO}}\right|^{2}+\left|E_{s}(t)\right|^{2}+2 \operatorname{Re}\left[E_{s}(t) E_{\mathrm{LO}}^{*} \exp \left(i\left(\omega_{s}-\omega_{\mathrm{LO}}\right) t\right)\right] \\
& P_{2}(t)=\left|E_{\mathrm{LO}}\right|^{2}+\left|E_{s}(t)\right|^{2}+2 \operatorname{Im}\left[E_{s}(t) E_{\mathrm{LO}}^{*} \exp \left(i\left(\omega_{s}-\omega_{\mathrm{LO}}\right) t\right)\right]
\end{aligned}
$$

Either one of these detected powers alone cannot be used to recover the information on the signal in the case where the difference frequency is less than the bit rate, because the information vanishes during the null of the difference frequency envelope. The information can be recovered from the two powers combined, as follows:

$$
E_{s}(t)=\frac{1}{2 E_{\mathrm{LO}}^{*}} \exp \left(-i\left(\omega_{s}-\omega_{\mathrm{LO}}\right) t\right)\left(\Delta P_{1}(t)+i \Delta P_{2}(t)\right)
$$

where $\Delta P_{1}=P_{1}-\overline{P_{1}}$, etc., and given that $\left(\omega_{s}-\omega_{\mathrm{LO}}\right)$ and the phase of $E_{\mathrm{LO}}$ are first determined during a lock acquisition phase and then continuously tracked. Equation (2) is derived from (1) assuming that the signal-squared term can be neglected, which is reasonable because the power of the LO was kept about $20 \mathrm{~dB}$ higher than the signal during the experiment. Note that (2) is different from the way information is usually obtained using phase diverse detection [4]. Previous implementations have been equivalent to squaring and adding $P_{1}$ and $P_{2}$, which discards the absolute phase of the signal, whereas (2) generates a complex (phasor) quantity. After digitizing the electrical 


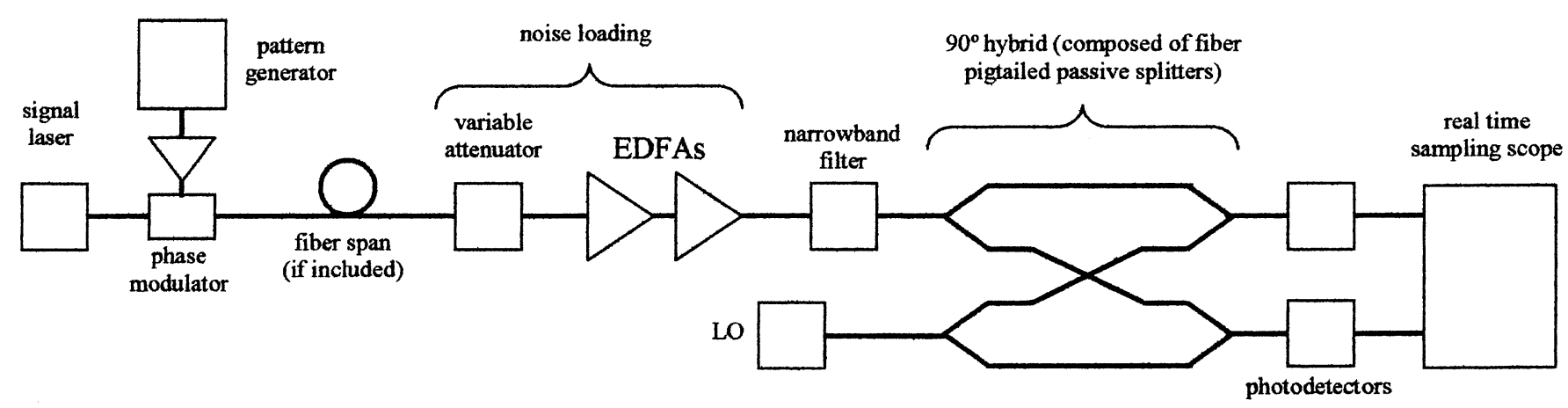

Fig. 1. Arrangement of experiment to demonstrate sampled coherent detection.

signals from the two photodetectors, (2) can be implemented in a DSP circuit. The LO and signal lasers do not have to be phase locked, and the DSP is doing the last stage of synchronous demodulation via a digital phase-locked loop. A similar operation in the field of digital radio is referred to as quadrature sampling. The result is that a continuous set of samples of the signal's complex electric field is available within the DSP. The transmitted information can be extracted directly, or signal processing can be applied first to compensate for impairments such as CD.

For any coherent detection method to be feasible, it is important to have a means of tracking the incoming signal state of polarization (SOP). Although not demonstrated here, this can be done by adding polarization diversity to the phase diverse arrangement [4]. This would require four photodetectors instead of two, but in fact, it is possible to perform polarization demultiplexing using the polarization diverse configuration, so the information carrying capacity of the signal is doubled also.

\section{EXPERIMENT}

To demonstrate the basic features of quadrature-sampled detection, a binary phase-shift keying (BPSK) transmitter was used, comprising a $\mathrm{LiNbO}_{3}$ phase modulator driven via a broad-band RF amplifier with a $2^{23}-1$ pseudorandom sequence at $10.66 \mathrm{~Gb} / \mathrm{s}$. A controlled amount of optical noise was added to the signal through the two erbium-doped fiber amplifiers shown in Fig. 1. The phase diverse configuration was made up of individual fiber pigtailed components. It was found that the phase difference between the arms drifted over time, and to obtain a phase difference of $\pi / 2$ several measurements were taken for each data point; in every case at least one was found subsequently to have close to $\pi / 2$ phase difference. The signal and LO lasers were external cavity lasers. The frequency difference between them drifted and was about $100 \mathrm{MHz}$. The SOPs of the signal and LO were approximately matched by manual manipulation of the fibers. The outputs of the photodetectors were recorded by a Tektronix TDS6604 real-time sampling oscilloscope, having 6-GHz front end bandwidth. This instrument was able to download continuous 4- $\mu$ s blocks from its two inputs in parallel at $20 \mathrm{GSa} / \mathrm{s}$, and then the data was transferred to a pesonal computer for offline processing. The oscilloscope + postprocessing was used because, although inexpensive when produced in volume, the cost of a dedicated DSP for this purpose would be prohibitive.

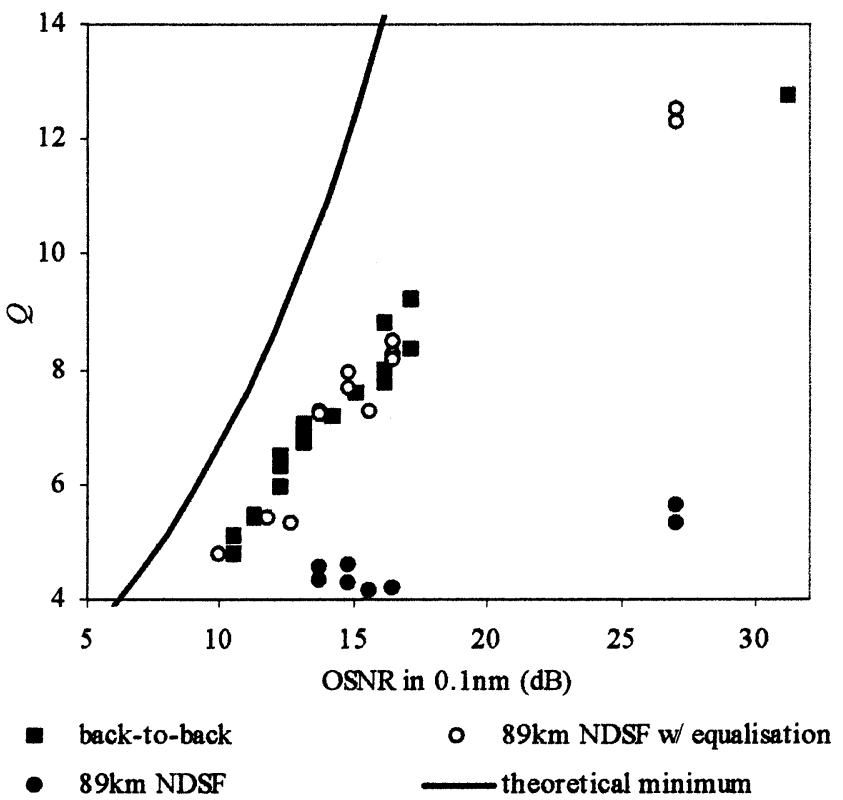

Fig. 2. Measured $Q$ factor versus OSNR.

Two sets of data of $Q$ factor versus OSNR were collected, for a noise-loaded back-to-back configuration and for transmission over $89-\mathrm{km}$ standard SMF with noise loading. The results are shown in Fig. 2. Each data point comes from a pair of traces $4 \mu \mathrm{s}$ in length. The measured data was processed as follows.

1) Each trace was convolved with a nine-point impulse response vector to reverse the nonflat frequency response of the detector + scope front end. The two impulse response vectors were determined by a simple adaptive process to give the best $Q$ factor on one of the noise-loaded measurements, and then the same vectors were applied to all measurements, with and without fiber.

2) The transmit clock $(10.66 \mathrm{GHz})$ and beat envelope (about $100 \mathrm{MHz}$ ) were recovered for the two channels.

3) The 20-GSa/s data was retimed using an interpolation technique to exactly two samples per bit, with alternate samples lying at the bit center. The same data would have been obtained if the A/D converters were clocked synchronously with the signal clock (and $2 \times$ the frequency). For the quadrature sampling process, only one sample per bit (at the bit center) is needed, and for CD compensation, two samples per bit are required. 
4) The two channels were combined according to (2), to give the complex envelope of the signal electric field.

5) For the measurements over fiber, an equalization filter was applied. The complex signal was convolved with the impulse response (inverse Fourier transform) of the CD transfer function $\exp \left(i\left(\frac{1}{2}\right) \omega^{2} \beta_{2} L\right)$, truncated to seven points. The value $\beta_{2} L=1.87 \times 10^{-21} \mathrm{~s}^{2}$ was used, equivalent to $89 \mathrm{~km}$ of standard SMF.

6) The $Q$ factor was calculated using the decision threshold technique on the alternate samples at the bit centers.

The signal processing operations above are all of the kind that can be executed by a high-speed DSP. For Step 5, the sevenelement vector was found to give the same results within $0.2 \mathrm{~dB}$ as a much longer vector (i.e., no truncation). Also, it was found that the simple convolution used (a fully nonrecursive digital filter) was as good as any mixed recursive-nonrecursive filter with the same number of taps.

The calculated $Q$ versus OSNR curves are shown in Fig. 2. The points for the back-to-back case indicate a very good sensitivity, requiring an OSNR of $10.5 \mathrm{~dB}$ for $Q=5$, which is only $2.7 \mathrm{~dB}$ from the theoretical minimum. This is believed to be the best ever reported back-to-back sensitivity at $10 \mathrm{~Gb} / \mathrm{s}$, and is $4-5 \mathrm{~dB}$ better than a typical intensity modulation-direction result. The theoretical curve in Fig. 2 is for BPSK obtained from the equations in [5], adapted to refer to OSNR instead of received power.

Also in Fig. 2 are the curves for transmission over $89-\mathrm{km}$ fiber, both without and with postdetection equalization. The equalization operation restores the sensitivity to the back-to-back values. The penalty due to $\mathrm{CD}$ is reduced from 5 to $0 \mathrm{~dB}$ at $Q=5$. As mentioned previously, the elimination of the $\mathrm{CD}$ penalty by postdetection signal processing can be achieved with coherent detection, but not direct detection.

\section{DISCUSSION AND CONCLUSION}

What has been shown in this letter is that the incorporation of new high-speed DSP technology means that coherent detection can be implemented with the high sensitivity associated with homodyne detection but without phase locking the LO laser. The detection of a BPSK encoded signal was demonstrated in a burst-mode experiment with offline processing of the data. Subsequent processing of the phasor representation of the signal is possible by the digital signal processor to compensate for propagation impairments, and this processing stage is equivalent to inserting an optical component having characteristics defined by the signal processing algorithm. A consequence of this optical domain-equivalent nature is that propagation impairments can be equalized completely. Penalty-free compensation of $1470 \mathrm{ps} / \mathrm{nm}$ of CD was shown, and a similar zero-penalty result can be expected for compensation of other impairments. The method also opens up the possibility of employing phase and polarization-encoded modulation formats cost effectively, which are in general more tolerant to fiber propagation effects and have higher spectral efficiency than the conventional formats used with direct detection.

\section{ACKNOWLEDGMENT}

The author thanks Tektronix for the loan of the oscilloscope and H. Yaffe and F. Moody for the loan of key items of equipment.

\section{REFERENCES}

[1] G. P. Agrawal, Fiber-Optic Communication Systems, 2nd ed. New York: Wiley, 1997.

[2] T. Mizuochi, K. Ouchi, T. Kobayashi, Y. Miyata, K. Kuno, H. Tagami, K. Kubo, H. Yoshida, M. Akita, and K. Motoshima, "Experimental demonstration of net coding gain of $10.1 \mathrm{~dB}$ using $12.4 \mathrm{~Gb} / \mathrm{s}$ block turbo code with 3-bit soft decision," presented at the Optical Fiber Communications Conf. (OFC 2003), Atlanta, GA, 2003, Paper PD21.

[3] G. Kanter, P. Capofreddi, S. Behtash, and A. Gandhi, "Electronic equalization for extending the reach of electro-absorption modulator based transponders," presented at the Optical Fiber Communications Conf. (OFC 2003), Atlanta, GA, 2003, Paper ThG6.

[4] L. G. Kazovsky, "Phase- and polarization-diversity coherent optical techniques," J. Lightwave Technol., vol. 7, pp. 279-292, July 1989.

[5] Y. Yamamoto, "Receiver performance evaluation of various digital optical modulation-demodulation systems in the $0.5-10 \mu \mathrm{m}$ wavelength region," IEEE J. Quantum Electron., vol. QE-16, pp. 1251-1259, Nov. 1980 